\title{
Should Noninvasive Ventilation Be Used for Treatment of Acute Cardiogenic Pulmonary Edema? A Cochrane Review Summary With Commentary
}

\author{
Dean R Hess
}

Background

Acute cardiogenic pulmonary edema is common in patients with heart disease. It can result in derangements in gas exchange and hemodynamics, leading to hypoxia and death. Standard medical care includes oxygen, diuretics, morphine, and an afterload reducer such as nitroglycerin. Noninvasive ventilation (NIV) and CPAP improve respiratory function by reducing the work of breathing and improving gas exchange. Moreover, the application of positive pressure to the airway increases pleural pressure, which decreases preload and afterload, thereby improving cardiac function. Thus, there is a strong physiologic rationale for the use of NIV or CPAP in the setting of acute cardiogenic pulmonary edema. Few would argue that positive pressure in the form of NIV or CPAP is preferable to endotracheal intubation. A Cochrane review evaluated the evidence to determine whether NIV reduces mortality and the need for endotracheal intubation. ${ }^{1}$

The purpose of this commentary is to discuss, with a respiratory care perspective, the published Cochrane Review, "Noninvasive Positive-Pressure Ventilation (CPAP or BiLevel NPPV) for Cardiogenic Pulmonary Oedema," under the direct supervision of Cochrane Heart Group. This Cochrane Corner is produced in agreement with Respiratory CARe by Cochrane Rehabilitation.

\section{What Is the Aim of This Cochrane Review?}

The aim of this Cochrane Review is to assess the effectiveness and safety of NIV compared to standard medical care for adults with acute cardiogenic pulmonary edema.

Dr. Hess is affiliated with Massachusetts General Hospital and Northeastern University, Boston, MA. He is also Managing Editor of Respiratory CARe.

Dr Hess has disclosed relationships with Ventec Life Systems, Daedalus Enterprises Jones and Bartlett, McGraw-Hill, and Up-To-Date.

This summary is based on a Cochrane Review previously published in the Cochrane Database of Systematic Reviews 2019;4:CD005351. doi:10.1002/ 14651858.CD005351.pub4 (See www.cochranelibrary.com for information.) Cochrane Reviews are regularly updated as new evidence emerges and in response to feedback, and Cochrane Database of Systematic Reviews should be consulted for the most recent version of the review. The views expressed in the summary with commentary are those of the Cochrane Corner author(s) and do not represent the Cochrane Library or Wiley.

\section{What Was Studied in the Cochrane Review?}

The population addressed in this review was individuals with cardiogenic pulmonary edema age $\geq 18 \mathrm{y}$. The interventions studied used nasal or oronasal noninvasive respiratory support in the form of NIV, CPAP, or both, with standard medical care. The intervention was compared to standard medical care alone for acute cardiogenic pulmonary edema without NIV or CPAP. The primary outcome studied was hospital mortality. Secondary outcomes were endotracheal intubation, treatment intolerance and failure, hospital and ICU length of stay, rates of acute myocardial infarction, arterial blood gases (at $1 \mathrm{~h}$ after intervention), and adverse event rates.

\section{Up-to-Dateness of the Cochrane Review?}

The review authors searched for studies published up to September 2018.

\section{What Are the Main Results of the Cochrane Review?}

The review included 24 studies involving 2,664 adults $>18$ y old with respiratory distress due to acute cardiogenic pulmonary edema who did not require immediate mechanical ventilation. When comparing noninvasive respiratory support with standard medical therapy alone, the review reported that:

- Noninvasive respiratory support in the form of NIV or CPAP might reduce hospital mortality (risk ratio [RR] $0.65,95 \%$ CI $0.51-0.82 ; 21$ trials with 2,284 participants; low quality of evidence).

- Noninvasive respiratory support in the form of NIV or CPAP probably reduces endotracheal intubation rates (RR 0.49 , 95\% CI $0.38-0.62 ; 20$ trials with 2,449 participants; moderate quality of evidence).

- There is probably little or no difference in the incidence of acute myocardial infarction with noninvasive respiratory support in the form of NIV or CPAP (RR 1.03,

Correspondence: Dean R Hess PhD RRT FAARC, Respiratory Care, Massachusetts General Hospital, Boston, MA 02114. E-mail: dhess@ mgh.harvard.edu.

DOI: $10.4187 /$ respcare. 07685 
95\% CI $0.91-1.16$; 5 trials with 1,313 participants; moderate quality of evidence).

- The authors are uncertain as to whether NIV or CPAP increases hospital length of stay (mean difference: $-0.31 \mathrm{~d}, 95 \% \mathrm{CI}-1.23$ to $0.61 ; 11$ trials with 1,714 participants; very low quality of evidence).

- In general, similar adverse events occur across noninvasive respiratory support in the form of NIV or CPAP and standard medical therapy (low quality of evidence).

\section{What Did the Authors Conclude?}

The authors concluded that this review provides support for clinical application of noninvasive respiratory support in the form of NIV or CPAP for acute cardiogenic pulmonary edema to improve outcomes such as hospital mortality and the need for endotracheal intubation. NIV and CPAP are safe interventions, showing similar adverse event rates as standard medical therapy alone. Future research is needed to determine whether specific subgroups have greater benefit and whether noninvasive respiratory support is beneficial for patients with hypercapnia.

\section{What Are the Implications of the Cochrane Evidence for Respiratory Care Practice?}

Noninvasive respiratory support in the form of NIV or CPAP is often considered standard practice for patients with acute cardiogenic pulmonary edema in the field, in the emergency department, in the critical care unit, and in the hospital ward. Respiratory therapists are usually integral to the application of this therapy in the hospital. This Cochrane Review provides relatively good evidence to support this practice, with a number-needed-to-treat of only 13 to prevent the need for intubation and only 17 to save a life. ${ }^{1}$

The findings of this review are consistent with recently published clinical practice guidelines by the European Respiratory Society (ERS) and the American Thoracic Society (ATS). ${ }^{2}$ Those guidelines, published in 2016, recommend either NIV or CPAP for patients with acute respiratory failure due to cardiogenic pulmonary edema (strong recommendation, moderate certainty of evidence). In the analysis accompanying these guidelines, noninvasive respiratory support in the form of NIV or CPAP decreased mortality (RR $0.80,95 \%$ CI $0.66-0.96$; moderate certainty) and decreased the need for intubation (RR $0.60,95 \%$ CI 0.44 0.80 ; low certainty). An increase in myocardial infarction was seen in the NIV group, but with a very low certainty of evidence (odds ratio $1.18,95 \%$ CI $0.95-1.48$ ). The committee felt that the desirable effects of noninvasive respiratory support in the form of NIV or CPAP outweighed the anticipated undesirable effects, but the recommendation does not apply to the subgroups of acute coronary syndrome or cardiogenic shock.

Both the Cochrane review ${ }^{1}$ and the ERS/ATS guidelines $^{2}$ combine results from studies of NIV and CPAP. Strictly speaking, that is not correct because the technical and physiologic effects of NIV and CPAP differ despite that they both apply positive pressure. The ERS/ATS guidelines ${ }^{2}$ state that the current evidence demonstrates significant benefit for both NIV and CPAP compared with standard care. Thus, there is insufficient evidence to recommend one approach over the other. According to the ERS/ATS guidelines, ${ }^{2}$ CPAP has advantages over NIV in terms of simpler technology, better synchrony, and potentially less expensive equipment. In a meta-analysis of CPAP and NIV for acute cardiogenic pulmonary edema, Weng et $\mathrm{al}^{3}$ reported no difference between NIV and CPAP on any clinical outcomes that were compared.

There should be little doubt about the evidence supporting the use of CPAP or NIV in the setting of acute cardiogenic pulmonary edema. This therapy should be considered standard practice in this setting.

\section{ACKNOWLEDGMENTS}

The author thanks Cochrane Rehabilitation and Cochrane Heart Group for reviewing the contents of the Cochrane Corner.

\section{REFERENCES}

1. Berbenetz N, Wang Y, Brown J, Godfrey C, Ahmad M, Vital FMR, et al. Non-invasive positive pressure ventilation (CPAP or bilevel NPPV) for cardiogenic pulmonary oedema. Cochrane Database Syst Rev 2019;4:CD005351.

2. Rochwerg B, Brochard L, Elliott MW, Hess D, Hill NS, Nava S, et al. Official ERS/ATS clinical practice guidelines: noninvasive ventilation for acute respiratory failure. Eur Respir J 2017;50(2):1602426.

3. Weng CL, Zhao YT, Liu QH, Fu CJ, Sun F, Ma YL, et al. Meta-analysis: noninvasive ventilation in acute cardiogenic pulmonary edema. Ann Intern Med 2010;152(9):590-600.

\section{(E) Cochrane}

